\title{
Diverse approaches to fighting infection in Northern Ireland
}

\author{
Nizam Damani, Trudy Reid, Jemima Keyes \\ Craigavon Area Hospital, Portadown, Co Amargh, Northern Ireland \\ doi:10.3396/ijic.V4s1.018.08
}

\begin{abstract}
Sustained media coverage, staff shortages and a number of outbreaks were only few of the challenges facing the infection control team at Craigavon Area Hospital in 2005. However, better communication along with the introduction of initiatives such as Bug Watch, the Wipe it Out campaign and a link worker training programme, assisted in the successful reduction of Clostridium difficile infection rates. Multi-modal intervention initiated by the infection control led to the reduction in the Methicillin Resistant Staphylococcus aureus (MRSA) bacteraemia rate. The hospital also achieved savings of $£ 42,000$ after reducing the inappropriate use of piperacillin/tazobactam.
\end{abstract}

\section{Introduction}

Craigavon Area Hospital (CAH) is a 466-bed acute hospital with adult and paediatric intensive care units (ICUs), and is also one of the regional cancer units. The hospital was built in 1972 and has the typical building design problems. These problems include a lack of isolation facilities, poor availability of hand washing sinks and inadequate bed spacing. The Trust's 96-bed Lurgan Hospital and 41-bed South Tyrone Hospital are both geriatric hospitals, and being much older than Craigavon Area Hospital present their own difficulties.

Craigavon Area Hospital's infection control team (ICT) was established in 1991 and currently consists of two infection control nurses (ICN) and one infection control doctor (ICD)/ microbiologist. The ICT is based at the Craigavon Area hospital and covers three hospitals in total.
The main objective of the team at the hospital is to provide leadership and direction to the senior management team. The function of the ICT is critical to helping the hospital achieve its goals, namely, the prevention and control of risks associated with hospital acquired infections.

\section{Method \\ The ICT participates in daily departmental meetings. The ICNs, ICD, microbiologists (both consultant and trainee) and the principal biomedical scientist (BMS) in microbiology attend these noon meetings. Clinical information gathered by the ICNs during the daily ward rounds and the microbiology information from the laboratory is discussed. The aim of the meeting is to communicate, discuss, educate and promptly act on all infection control and other issues on a daily basis.}




\section{Microbiology discussion group}

Since 1991, the department of microbiology has facilitated a discussion group, wherein three evening meetings are held each year. The meeting is attended by ICTs, consultant microbiologists, a consultant in communicable disease control, biomedical scientists, infection control nurses from neighbouring hospitals, clinical consultants, junior medical staff and laboratory administrative staff.

The aim of the meeting is to educate, enthuse and promote team building amongst the various staffs to achieve effective delivery of service. Topics discussed last year were: C. difficile, MRSA in community, and avian and pandemic flu. The meeting is CPDaccredited and followed by dinner to allow informal discussion and team building.

\section{Infection control link person}

The department has also established a formal infection control link person (ICLP) training programme. This year 98 front line staff including senior nurses, PAMS, radiotherapists, physiotherapists and bed managers have attended the training sessions. The training includes both formal presentations and practical training sessions. The ICLP meetings are held on a monthly basis with the aim to communicate and educate. Issues arising from audit and observations at ward and departmental level are discussed.

\section{Meetings}

The Chief Executive has a very keen interest in infection control and meets with the ICT on a bimonthly basis. All issues relating to infection control are discussed. The meeting is attended by the medical director, director of nursing and quality and director of health estates. Key decisions are made at this meeting and relevant issues are highlighted to the hospital board by the chief executive.

\section{Infection control committee}

The local infection control committee (LICC) is also unique in that it is composed of medical director, director of nursing and quality, clinical directors, director of health estates, Consultant in Communicable Disease Control/Public Health Doctor (CCDC) and occupational health department. In addition, the ICT also attend the area ICC chaired by the CCDC. Minutes of the meeting are copied to the Chief Executive. The
ICD is also the current Clinical Director of pathology services and presents infection control issues to senior management including the clinical governances committee and the hospital board. To raise profile and awareness in infection control, in 2005, ICT gave presentations at the: Trust Annual General Public meeting, Southern Health and Social Service Council, Craigavon Trust board, non-executive directors of the Southern Health and Social Services board.

Through the hospital's local public relations offices, the team has also been involved in regular press briefings to keep the public aware of infection control in a positive way.

\section{Results}

\section{C. difficile}

Mandatory surveillance of $C$. difficile in Northern Ireland started in January 2005 and when the quarterly figures were fed back to the ICT in June 2005, the ICT found that two of its hospitals had the highest incidence of $C$. difficile within the province.

This prompted the ICT to inform senior management and convene multi-disciplinary meetings involving senior medical and nursing staff. Various measures were taken including education of staff at ward level, emphasis on hand washing, environmental cleaning, review and audit of antibiotic guidelines and new protocols for sending faecal specimen.

The team also decided to do a retrospective audit of all patients with $C$. difficile. Multi-modal interventions have proved very successful and this has resulted in sustained reduction of $C$. difficile rates over the following months.

\section{MRSA surveillance}

$\mathrm{CAH}$ has been participating in the Department of Health mandatory surveillance of MRSA since 2001. Multi-modal intervention initiated by the ICT has seen a reduction in the MRSA bacteraemia rate in 2004-05.

\section{Piperacillin/tazobactam audit}

Through the Drugs and Therapeutic Committee, it was highlighted that there has been a substantial increase in the use of piperacillin/tazobactam in both hospitals. It was decided that the ICD would carry out an audit for six weeks during February and March 2005. The 
audit data were analysed and recommendations were fed back to the appropriate clinicians. As a result of this audit, Craigavon hospital has saved $£ 42,000$ compared with the previous three quarters.

\section{Wipe it Out}

On 2 November 2005 the Craigavon Hospital Group Trust was chosen by the Royal College of Nursing as their pilot site to launch the Wipe it Out campaign in $\mathrm{N}$ Ireland. This campaign generated very positive publicity for the hospital and the event was covered by the radio, television and newspapers.

\section{Bug watch}

On 21 September 2005, Craigavon Area Hospital also participated in the Bug watch survey. Members of a visiting team from the Southern Health and Social Services Council were impressed with the standard of IC practices and summarised their findings stating: "The Council was most satisfied with the results of the Bug watch survey at Craigavon hospital."

\section{WHO Global Patient Safety Challenge}

On 13 October 2005, Craigavon was the only hospital in N Ireland to participate in the launch of World Health Organization's Global Patient Safety Challenge Clean care is Safer Care. This occasion was marked by setting up a stand with educational material for the public at the hospital foyer. Education sessions on hand hygiene throughout the hospital were conducted. This event was covered by the local press.

Dr N Damani has also been appointed as a member of the core group in Geneva to help drive the Global Patient Safety Challenge at a global level.

\section{Hand hygiene}

With the introduction of a new hand gel, the ICT has helped organise the training sessions on hand hygiene for all staff. In total, 16 sessions were organized between June and August 2005. The sessions were attended by 402 healthcare workers (doctors, nurses, physiotherapists, radiographers, speech therapists etc) from all three hospitals.

\section{Audit of Sharp disposal}

In June 2005, an audit of sharps disposal was carried out. The findings were presented to the IC Link person training sessions. As a result of this audit, reviews of provisions of sharp boxes were carried out and trolley brackets were provided to secure boxes. This was followed by training session at ward level to ensure compliance with the hospital sharp disposal policy.

\section{Outbreaks}

\section{Norovirus outbreak}

During 2005, the ICT investigated and successfully controlled nine outbreaks of Norovirus infection in a variety of wards from two hospitals.

\section{Outbreak of endopthalmitis}

In October 2005, ICT were informed of an outbreak of endopthalmitis after cataract surgery. The surgeries were performed over two weeks by a foreign independent healthcare provider as a part of waiting list initiative. At a very short notice, the ICT carried out a thorough investigation to establish the cause of the outbreak. The ICT worked tirelessly over the Christmas/New Year holiday period to complete the investigation and prepared a report for submission to the external assessor appointed by the Southern Board. This incident attracted intense media attention (TV, radio and newspapers). The ICT found this experience uniquely challenging.

\section{MRSA outbreak}

In October 2005, the ICT was responsible for the management of an outbreak of MRSA in the neonatal ICU. This was the first outbreak of MRSA in the neonatal unit since the hospital was opened in 1972. Preterm babies were affected with septicaemias or pneumonias. ICT successfully controlled this outbreak. As a result of this outbreak IC practices in the unit were reviewed, onsite education sessions were given and other infection control and environmental issues were addressed in order to prevent a recurrence.

\section{Environmental cleaning}

During 2004, the ICT and the cleaning team of the hospital carried out an extensive review of the cleaning procedure. Funding for this project was awarded by the $\mathrm{DH}$ after submission of a business case for the project by the hospital facility management and ICT. During the review, it was noticed that cleaning in the hospital was carried out in an inconsistent manner.

During 2005, the project findings were reviewed and this resulted in the formulation of a protocol for 
the decontamination of all high touch surfaces with a hypochlorite-based detergent preparation. This procedure has now been uniformly implemented and substantial improvements have been noted.

\section{Operating theatres}

In July 2005, the health estates department informed the ICT that a survey of the 33-year old operating theatre revealed major deficiencies in the ventilation system.

This initiated a flurry of activities. Measures taken included:

- risk assessment of surgical procedures;

- audit of theatre practices;

- testing of ventilation system;

- seeking independent advice from the Health Protection Agency in London; and

- preparing a business case to the $\mathrm{DH}$ for additional funding to rectify the problem.
The ICT took several measures to minimise the risk of surgical site infections that included:

- referral of all high risk procedures to other hospital;

- implementation of strict theatre discipline \& traffic control;

- estates work to improve both the air flow and air exchange;

- microbiological examination of theatre testing on several occasions; and

- housekeeping issues.

As a result, there have been significant improvements in the ventilation system, traffic control and theatre practices. A business case has been submitted to the DH major capital development plan.

\section{Research}

Is your phone bugged?

In order to assess microbial contamination on the

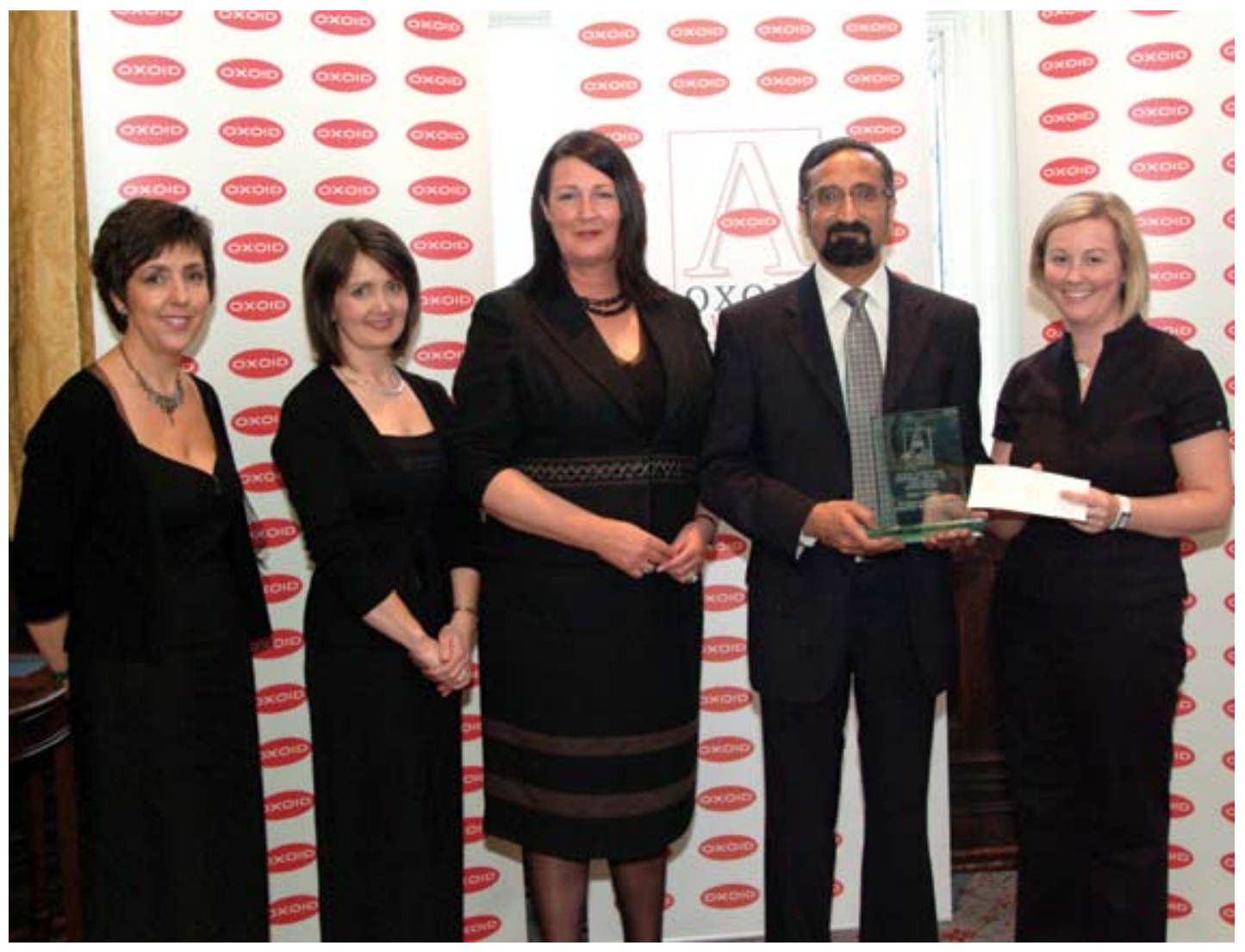

The team received the first prize in 2005/06 OXOID awards 
mobile phones amongst health care workers, the ICD conducted a study by sampling 102 mobile phones from doctors and nurses in Craigavon Area Hospital and found $2 \%$ carriage of MRSA. ${ }^{1}$

\section{Conclusions}

The year 2005 was the most hectic in the team's 14-year history. A few challenges the team faced included: the highest incidence of $C$. difficile within the province in the first quarter; outbreaks of Norovirus; MRSA in NNU and ICU; post operative endopthalmitis and; problems with the ageing operating theatres ventilation system.

Despite this, the team has managed to formally establish a Link Person Training Programme and is the first hospital to participate in the Wipe it out campaign. It is the only hospital within $\mathrm{N}$ Ireland to launch the WHO Global patient safety challenge programme.

In addition to that, the team has successfully managed to reduce both $C$. difficile and MRSA infection rates and achieved savings of $£ 42,000$ reduction from the inappropriate use of piperacillin/tazobactam. This was achieved despite the staffing shortage with one full time ICN on sick leave for most of the year (from October 2004 to September 2005) and minimal secretarial support due to long term sickness not to mention the sustained media attention from the television, radio and newspapers.

The team not only met all the challenges successfully but also achieved sustained and real improvement in various areas. These improvements ensure that the hospital environment is safe for the patients, staff and visitors. This was achieved through a dynamic team effort throughout the year with a clear focus on the improvement of quality for our patients.

\section{Reference}

1. Brady RRW, Wasson A, Stirling I, McAllister C, Damani NN. Is your phone bugged? The incidence of bacteria known to cause nosocomial infection on healthcare workers' mobile phones. J Hosp Infect 2006; 62 (1): 123-125. 